\title{
Experimental evidence for large dynamic effects on the plasmon dispersion of subwavelength metal nanoparticle waveguides
}

\author{
A. Femius Koenderink * René de Waele, Jord C. Prangsma, and Albert Polman \\ Center for Nanophotonics, FOM Institute for Atomic and Molecular Physics, Kruislaan 407, 1098 SJ Amsterdam, The Netherlands \\ (Dated: Published as Phys. Rev. B 76, 201403(R) (2007). [Received July 18, 2007, published November 13, 2007)])
}

\begin{abstract}
We present angle and frequency resolved optical extinction measurements to determine the dispersion relation of plasmon modes on Ag and Au nanoparticle chains with pitches down to $75 \mathrm{~nm}$. The large splitting between transverse and longitudinal modes and the band curvature are inconsistent with reported electrostatic near-field models, and confirm that far-field retarded interactions are important, even for $\lambda / 5$-sized structures. The data imply that lower propagation losses, larger signal bandwidth and larger maximum group velocity then expected can be achieved for wave vectors below the light line. We conclude that for the design of optical nanocircuits coherent far-field couplings across the entire circuit need to be considered, even at subwavelength feature sizes.
\end{abstract}

A fundamental limit to the realization of sub-wavelength (sub- $\lambda$ ) optical devices is that the interaction strength of dielectric objects with light vanishes as the objects gets smaller: 1.2 Plasmonics may allow to overcome this inherent limitation of dielectrics by packing the large polarizability of free electron resonances into a small physical volume ${ }^{2-4}=$ In this framework plasmon particle arrays have been proposed as an ideal platform that combines the ease of controlled nanofabrication with the prospects of creating, e.g., ultrasmall antennas to efficiently harvest, enhance and emit optical power,, 5.6 as well as a toolkit for nanophotonic circuits? ${ }^{7}$ Thus plasmon chains may act as sub- $\lambda$ width waveguides, waveguide bends, signal splitters, and filters $\stackrel{-8-11}{=}$ As a parallel development, sub- $\lambda$ arrays of scatterers with magnetic rather than electric resonances have recently gained tremendous interest for developing optical metamaterials. $\underline{\underline{12}}$

Pioneering experiments have focused on qualitative understanding of the resonance splitting and mode structure in plas-

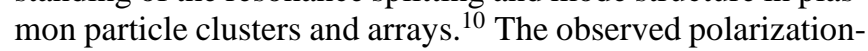
dependent resonances in linear (1D) particle chains, for instance, correlated well with trends anticipated from a simple near-field quasi-electrostatic model for the chain dispersion relation.,11 This 'quasistatic' model, which is valid on deep sub- $\lambda$ length scales, also formed the basis for forecasting the functionality of more complex structures, such as plasmon chain splitters and multiplexers. $\stackrel{79,13}{ }$ Very recently, however, several groups have developed electrodynamic models that predict large quantitative and qualitative deviations from the quasistatic insights. ${ }^{14}-18$ If these deviations indeed occur, a redevelopment of general design rules for complex nanophotonic circuits is required to include electrodynamic effects even on small length scales.

Sofar, quantitative experiments to discriminate between quasistatic and electrodynamic predictions at sub- $\lambda$ spacings have not been reported. In this Letter we present angle and frequency resolved optical extinction experiments on many arrays of $\mathrm{Ag}$ and $\mathrm{Au}$ nanoparticles at various particle sizes and sub- $\lambda$ pitches down to $\lambda / 5$. Sub- $\lambda$ plasmon arrays are an interesting system to test for dynamic effects. Recently, large modifications were predicted for the dispersion of modes in 1D plasmon particle chains and, equivalently, in 1D magnetic split ring resonator arrays $\frac{19}{9}$ For instance, the deviations include a much larger $k=0$ splitting between the longitu- dinal and transverse modes, and a polariton splitting of the transverse dispersion branch at the crossing with the vacuum dispersion relation (light line) $\stackrel{15-18}{-18}$ For sub- $\lambda$ waveguiding these models further imply a large increase in group velocity and decrease in propagation loss compared to electrostatic predictions. $\underline{17}$ The dispersion relations measured in our experiment agree well with the recent electrodynamic models, and deviate strongly from quasistatic predictions down to pitches as small as $\lambda / 5$. As a consequence we anticipate that design proposals for sub- $\lambda$ nano-optical circuits at currently realistic sizes $(\sim 50 \mathrm{~nm}$ pitch) can not be based on quasistatic analysis, $\stackrel{7}{?}$ because coherences and coupling across the full structure will dominate the optical performance.

We determine the nanoparticle chain dispersion relation above the light line by far-field extinction measurements on $\mathrm{Ag}$ and $\mathrm{Au}$ nanoparticle arrays prepared on glass using electron-beam lithography. After physical vapor deposition of $\mathrm{Ag}$ or $\mathrm{Au}$ and resist liftoff, we obtained linear arrays of particles of $50 \mathrm{~nm}$ height, at pitches of $d=75,100,120$ and $150 \mathrm{~nm}$ and with radii varied between $r=25$ and $55 \mathrm{~nm}$, as determined by scanning electron microscopy (SEM, cf. Fig.1(A,B)). The estimated error in determining $r$ is $2 \mathrm{~nm}$. For each pitch the particle radii are below $r / d=0.37$. We have fabricated square fields containing parallel particle chains (chain length $60 \mu \mathrm{m}$ ), with randomly varying inter-

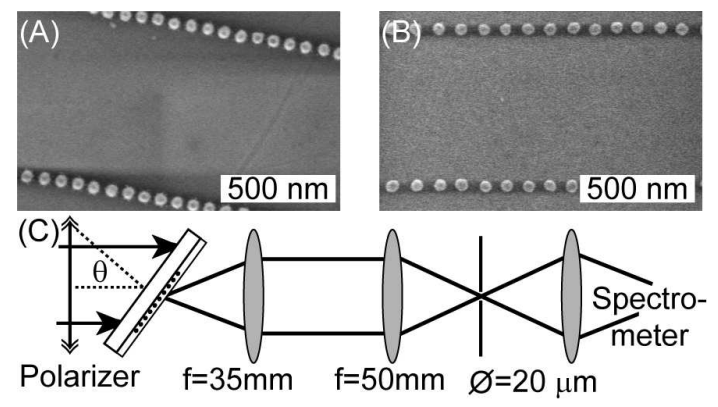

FIG. 1. (A, B) Scanning electron micrographs of particle arrays on glass with $r=25 \mathrm{~nm}, d=75 \mathrm{~nm}(\mathrm{~A}, \mathrm{Au})$ and $r=25, d=100 \mathrm{~nm}$ (B, Ag). (C) Overview of the angle-dependent transmission setup. A collimated beam illuminates a large sample area. The sample angle is varied. Light is collected from a small spot on the sample (collection $\mathrm{NA}=0.2$ ). 


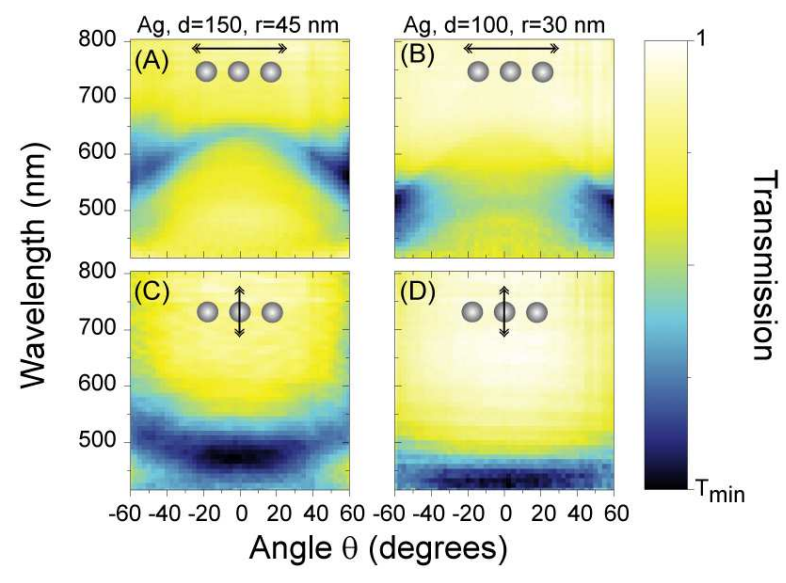

FIG. 2. (Color online) Transmission as a function of angle and wavelength for longitudinal $(\mathrm{A}, \mathrm{B})$ and transverse polarization $(\mathrm{C}, \mathrm{D})$ for Ag arrays with $r=45, d=150 \mathrm{~nm}(\mathrm{~A}, \mathrm{C})$ and $r=30, d=100 \mathrm{~nm}$ $(\mathrm{B}, \mathrm{D})$. The color scale runs from $T_{\min }=0.65$ in $(\mathrm{A}, \mathrm{C})$ and $T_{\min }=$ 0.80 in $(\mathrm{B}, \mathrm{D})$.

chain spacing (minimum $700 \mathrm{~nm}$, mean $1 \mu \mathrm{m}$ ). The large inter-chain spacing ensures that coupling between chains is negligible, while the random variation suppresses grating effects that occur for periodic arrangements. Finally, we spincoat the samples with a $100 \mathrm{~nm}$ layer of PMMA to ensure that the particle chains are embedded in a homogeneous dielectric environment. To determine the dispersion relation we use a wavelength-resolved transmission setup (see Fig. 1(C)) in which the sample is mounted on a rotation stage (axis perpendicular to the chains, along the PMMA/glass interface) that gives access to incident angles from $\theta=-60^{\circ}$ to $+60^{\circ}$. The chains are illuminated by a collimated white-light beam (divergence $\sim 5^{\circ}$ ) from a fiber-coupled incandescent source, which illuminates a large (mm-size) area on the sample. Using a pinhole on the transmission side, only the transmitted intensity from a $\sim 15 \mu \mathrm{m}$ spot (associated $\Delta k / k \sim 0.09$ (i.e., $\left.5^{\circ}\right)$ ) on the sample is collected by a cooled Si-CCD coupled spectrometer. The transmission is obtained by normalizing the transmitted intensity to that recorded from an unpatterned substrate at the same angle. Using a broadband polarizer, we select the incoming polarization to be either transverse to the chains, or longitudinal (p-polarization). For nonzero $\theta$, the ppolarization also acquires a field-component transverse to the chain.

Figure 2 shows transmission spectra for the full angular range for $\mathrm{Ag}$ chains of pitch $150 \mathrm{~nm}$, and radius $45 \mathrm{~nm}$ (Fig 2 (A,C)), and for a smaller pitch of $100 \mathrm{~nm}$ and radius $30 \mathrm{~nm}(\mathrm{Fig} 2(\mathrm{~B}, \mathrm{D}))$. At normal incidence $\left(\theta=0^{\circ}\right)$, a band of extinction around $\lambda=500 \mathrm{~nm}$ for $d=150 \mathrm{~nm}(470 \mathrm{~nm}$ for $d=100 \mathrm{~nm}$ ) is observed for transverse polarization, and at $\lambda=625 \mathrm{~nm}(550 \mathrm{~nm}$ for $d=100 \mathrm{~nm})$ for longitudinal polarization. The redshift of modes for larger particles is consistent with the well-known single-particle resonance shift with particle size $\stackrel{2,20}{2}$ The occurrence of two bands shifted to either side of the single particle resonance is consistent with earlier reports on the $\theta=0^{\circ}$ extinction of nanoparticle chains $\frac{10}{.}$ Qualitatively this splitting corresponds to the excitation of col- lective modes in the chain of dipole scatterers: the transverse mode is blue shifted due to the antiparallel orientation of each dipole with the field of its neighbors, while the longitudinal mode is red shifted as each dipole is aligned with the field of its neighbors. For increasing angle of incidence, the two branches have opposite curvature, both shifting towards the single particle resonance.

The spectral dependencies of the extinction branches evident in Fig. 2 are qualitatively consistent with both the quasistatic model and full dynamic calculations for the dispersion relation of plasmon chain excitations. In order to quantitatively compare the data with the two models, we determine the transmission minima from a Gaussian fit to the transmission spectrum (plotted in the frequency domain) for each angle. In figure 3, we plot the resulting center frequencies taken from Fig. 2] B,D) as a function of $\left|\mathbf{k}_{\|}\right|=2 \pi / \lambda \sin (|\theta|)$, i.e. the wave vector component of the incident beam along the chain. The measurements reach up to $k_{\|}=0.86 \mathrm{\omega} / \mathrm{c}$, or up to $60 \%$ of the light line in the medium embedding the particles. To appreciate the large width of the extinction resonances, the bandwidth at $1 / e$ height of the fitted Gaussians is shaded in the diagram. The typical $1 / e$ full width is $2500 \mathrm{~cm}^{-1}(\sim 60$ $\mathrm{nm}$ ). First, we compare to the generic quasistatic point-dipole prediction for the nanoparticle chain dispersion relation: ${ }^{9}-11$

$$
\omega^{2}=\omega_{0}^{2}\left[1+\left(\frac{r}{d}\right)^{3} \sum_{j=1}^{\infty} \kappa_{T, L} \frac{\cos (j k d)}{j^{3}}\right] .
$$

Here $\omega_{0}$ is the single-particle resonance frequency, and $\kappa_{T}=$ 2 for transverse, and $\kappa_{L}=-4$ for longitudinal modes 21 We assume spherical particles with $r / d$ taken from SEM data to obtain the quasistatic prediction in Fig. 3 (dotted curves). There are two striking discrepancies between the data and the quasistatic model. First, the splitting at normal incidence $\left(k_{\|}=0\right)$ between the two branches is a factor two to three larger in the data than in the quasistatic model. The quasistatic model even falls outside the broad width of the extinction peaks. Secondly, the quasistatic model predicts that the transverse and longitudinal branch cross at $k d=0.46 \pi$ independent of $r / d$ at a frequency equal to the single-particle resonance. No sign of this crossing is observed in the data. It seems surprising that such discrepancies between data and the quasistatic model haven't been noted in earlier studies $\stackrel{10}{i}$ These studies focused on $k=0$ only, without investigating nonzero scattering angles. On the basis of $k=0$ data only, one might assume that a larger splitting is due to an error in $r / d$. For our data, this would imply an unlikely $40 \%$ error in estimating $r / d$. However, even if one would scale $r / d$ to match the $k_{\|}=0$ splitting, the quasistatic model would still not be consistent with the full angle-dependent data set: the presence of the band crossing at $k d=0.46 \pi$ would impose a much larger curvature of both bands than observed in our data.

The fact that the quasistatic model does not describe the data could be due to several approximations: The quasistatic model ignores dynamic effects, multipole effects, the presence of the PMMA-air interface, and particle anisotropy. Based on Ref. 11 we conclude that multipole effects do not resolve 


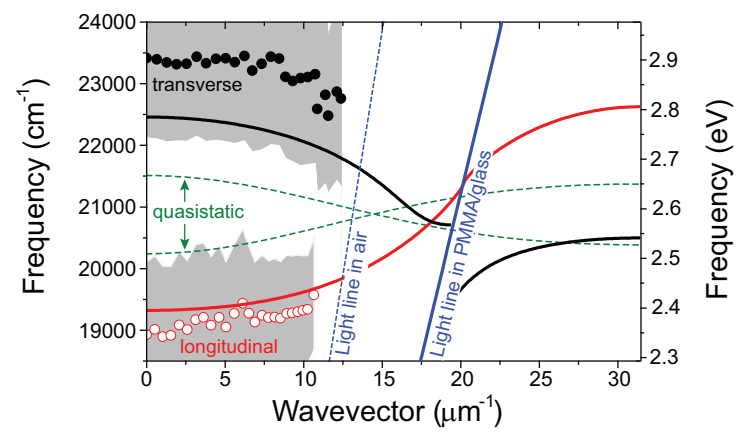

FIG. 3. (Color online) Symbols: frequency (in $\mathrm{cm}^{-} 1$ (left axis) or $\mathrm{eV}$ (right axis)) of minimum transmission versus wave vector for transverse and longitudinal polarization in Fig. 2 (B,D), i.e. the $d=100 \mathrm{~nm}, r=30 \mathrm{~nm} \mathrm{Ag}$ particles. Shaded areas: 1/e bandwidth of the transmission minima. Straight lines: light lines in air and in the embedding medium. Thick (thin) curves: dynamic (static) prediction.

the discrepancy between the quasistatic model and our data: multipole effects do not alter the quasistatic dispersion relation for $r / d<0.35$, a criterion satisfied in our experiment $(r / d=0.3 \pm 0.02)$. For larger $r / d$ multipole effects in fact reduce the quasistatic $k=0$ splitting, and shift the band crossing to even smaller wave vectors, 11 inconsistent with the absence of a crossing in our data. To study the effect of the dielectric interface, we have analyzed the quasistatic model for dipoles near a dielectric interface using image dipole theory. 1 Within this model the image dipoles have a weaker dipole moment by a factor $\left(\epsilon_{m}-1\right) /\left(\epsilon_{m}+1\right)$ (with $\epsilon_{m}$ the embedding dielectric constant) and are located at a distance of $150 \mathrm{~nm}$, i.e., further than the array pitch. As a consequence the estimated increase in splitting is less than $15 \%$, i.e., much less than the 2 to 3 -fold enhancement in the experiment. Regarding anisotropy, the particles for which data is reported in Fig. 3 are mildly oblate (height $50 \mathrm{~nm}$ and diameter $60 \mathrm{~nm}$ ). At $\theta=0^{\circ}$, both polarizations used in the experiment are along equivalent axes of the single-particle polarizability tensor, so that an enhanced splitting is not due to excitation of distinct singleparticle resonances. In addition, we calculated the polarizability tensor (including dynamic depolarization shifts ${ }^{3,20}$ ). The resonance relative to that of a $r=30 \mathrm{~nm}$ spherical particle is only shifted by $4 \mathrm{~nm}$, and the on-resonance polarizability along the long axes is in fact $10 \%$ smaller. Within the quasistatic model, particle anisotropy is hence expected to reduce the splitting, rather than explaining the observed enhancement.

Having excluded that modifications to the quasistatic model due to multipole effects, interface corrections or particle anisotropy can explain the dispersion relation observed in the experiment, we now compare the data to a full electrodynamic point-dipole model 17 that includes Ohmic damping, radiation damping, and depolarization shifts in the single-particle polarizability ${ }^{20}$, as well as all terms in the dipole field. Within this model the dispersion relation has recently been calculated perturbatively ${ }^{15}$, and self-consistently for finite ${ }^{16}$ and infinite arrays $\stackrel{17,18}{=}$ Using a dielectric model for silver that is a mod-

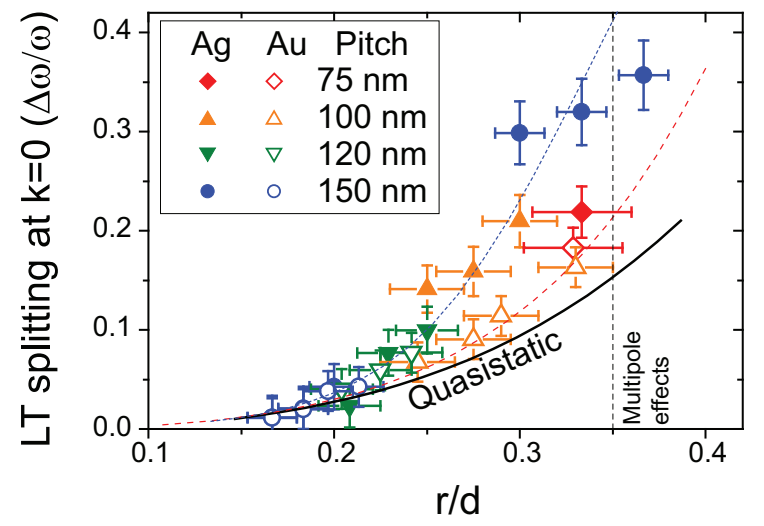

FIG. 4. (Color online) Symbols: Relative splitting between transverse and longitudinal branch at $k=0$ versus $r / d$ for several pitches $d$ as indicated for $\mathrm{Ag}$ and $\mathrm{Au}$ arrays. Black curve: quasistatic prediction for Ag arrays. Colored curves: dynamic predictions for $d=75 \mathrm{~nm}$ (Ag, dashed) and $d=150 \mathrm{~nm}$ (Ag, dotted). Multipole effects set in beyond the dashed vertical. ${ }^{11}$

ified Drude fit ${ }^{22}$ to tabulated data, $\stackrel{23}{=}$ and taking $r$ and $d$ from SEM observations, we plot the self-consistent infinite array model as solid curves in Fig. 3. This model predicts a band splitting at $k=0$ that is approximately twice the splitting in the quasistatic model for the same geometrical parameters, as well as a band curvature for wave vectors away from $k=0$ that is in much better agreement with the experimental data. Keeping in mind that the dynamic model has no adjustable parameters, the reasonable correspondence with the data is a strong indication that retardation effects and far-field coupling are indeed determining the dispersion relation for $d=100 \mathrm{~nm}$ silver nanoparticle chains. The remaining deviation from the dynamic model may yet again be due to the small particle anisotropy, the PMMA-air interface, or multipole effects ${ }^{14}$ the influence of which is much more difficult to estimate quantitatively for electrodynamic rather than quasistatic models.

Based on the data in Figs. 2, 3 we conclude that the large splitting between the transverse and longitudinal branch at normal incidence compared to the quasistatic prediction is a good indicator for the relevance of far-field effects. Figure 4 shows the measured relative splitting $\Delta \omega / \omega$ for many combinations of particle radius $r$ and pitch $d$ plotted against $r / d$, both for $\mathrm{Ag}$ and Au particle arrays. In the quasistatic limit, the splitting is simply proportional to $(r / d)^{3}$ (see Eq. (1)). In the dynamic model, the splitting depends on $r$ and $d$ separately as is clear from the dynamic predictions for the smallest $(d=75 \mathrm{~nm})$ and largest $(d=150 \mathrm{~nm})$ pitch used in our experiments (dashed/dotted in Fig. 47). Figure 4 demonstrates that for $\mathrm{Ag}$ particles the splitting is generally a factor $\sim 2$ larger than quasistatic theory predicts, in agreement with the dynamic model. For Au particles, the difference is not as large, which may explain why previous studies did not resolve deviations from quasistatic theory $\stackrel{10}{ }^{0}$ Still, the observed splittings for Au systematically exceed the quasistatic prediction. We attribute this smaller effect for gold to the much lower albedo ( $\leq 20 \%$ for $r=25 \mathrm{~nm} \mathrm{Au}$, compared to $\sim 80 \%$ for Ag) $\stackrel{2}{=}$ Since low-albedo particles radiate less strongly, far-field 
corrections to the dispersion relation will be less important.

In conclusion, the experiment reported in this Letter shows that the dispersion relation for plasmon modes on sub- $\lambda$ metal chains is strongly modified by far-field interactions, even for pitches as small as $d=75 \mathrm{~nm}$ or $\sim \lambda / 5$. This experiment thus confirms recent models ${ }^{15-18}$ that overturn the quasistatic view on plasmon chains. ${ }^{9,11}$ The next challenge is to address wave vectors below the light line, for which propagation distances up to $5-10 \mu \mathrm{m}$ at group velocities around $0.3 \mathrm{c}$ are feasible. 17 These propagation distances far exceed initial estimates, which were based on the damping rate of single particles ${ }^{9,10}$ Below the light line, the damping rate is strongly reduced since far-field destructive interference suppresses all radiative loss. It will be a challenge to excite these wave vectors selectively: local excitation, e.g. at a waveguide entrance, will excite wave vectors both below and above the light line. Recent simulations confirm that retardation effects are very important for local excitation of finite sub- $\lambda$ plasmon chains, giving rise to a complex and strongly frequency dependent response.$\frac{5}{}$ However, interpretation in terms of superpositions of modes above and below the light line is nontrivial, since depending on the excitation either the dispersion relation for real $k$ and complex $\omega$, or for complex $k$ with real $\omega$ applies, as discussed in Ref. 17. As the complexity is increased to include 2D clusters, we expect far-field effects to become even stronger. ${ }^{14}$ Therefore, our work implies that an essentially quasistatic electric circuit-design approach ${ }^{7}$ to nanophotonics is only applicable to structures of total size below $\sim 50 \mathrm{~nm}$, i.e., a scale at which fabrication of several coupled components is extremely challenging. Sub- $\lambda$ photonic structures at the current fabrication limit will always require a fully electrodynamic optimization of the coherent coupling between all building blocks: far-field interference is key to optimize the functionality and reduce the loss.

We thank L. Kuipers for loan of equipment and H.A. Atwater for fruitful discussions. This work is part of the research program of the "Stichting voor Fundamenteel Onderzoek der Materie (FOM)," which is financially supported by the "Nederlandse Organisatie voor Wetenschappelijk Onderzoek (NWO)". It was also supported by "NanoNed", a nanotechnology program funded by the Dutch Ministry of Economic Affairs.
* f.koenderink@amolf.nl

1 J. D. Jackson, Classical electrodynamics (Wiley, New York, 1975).

2 C. F. Bohren and D. R. Huffman, Absorption and scattering of light by small particles (Wiley, New York, 1983).

${ }^{3}$ K. L. Kelly, E. Coronado, L. L. Zhao and G. C. Schatz, J. Phys. Chem. B 107, 668 (2003).

${ }^{4}$ For a review, see W. L. Barnes, A. Dereux, and T. W. Ebbesen, Nature 424, 824 (2003).

5 J. V. Hernández, L. D. Noordam, and F. Robicheaux, J. Phys. Chem. B 109, 15808 (2005)

${ }^{6}$ R. de Waele, A.F. Koenderink, and A. Polman, Nano Lett 7, 2004 (2007).

7 N. Engheta, A. Salandrino, and A. Alù, Phys. Rev. Lett. 95, 095504 (2005); N. Engheta, Science 317, 1698 (2007).

${ }^{8}$ M. Quinten, A. Leitner, J. R. Krenn, and F. R. Aussenegg, Opt. Lett. 23, 1331 (1998).

9 M. L. Brongersma, J. W. Hartman, and H. A. Atwater, Phys. Rev. B 62, R16356 (2000).

${ }^{10}$ S. A. Maier, M. L. Brongersma, P. G. Kik, and H. A. Atwater, Phys. Rev. B 65, 193408 (2002); S. A. Maier, P. G. Kik, and H. A. Atwater, Appl. Phys. Lett 81, 1714 (2002).

11 S. Y. Park and D. Stroud, Phys. Rev. B 69, 125418 (2004).
12 D. R. Smith, J. B. Pendry, and M. C. K. Wiltshire, Science 305, 788 (2004).

13 L. Dobrzynski, A. Akjouj, B. Djafari-Rouhani, J. O. Vasseur, M. Bouazaoui, J. P. Vilcot, H. Al Wahsh, P. Zielinski, and J. P. Vigneron, Phys. Rev. E 69, 035601(2004).

14 L. Zhao, L. Kelly, and G. C. Schatz, J. Phys. Chem. B 107, 7343 (2003).

15 D. S. Citrin, Opt Lett. 31, 98 (2005); C. R. Simovski, A. J. Viitanen, and S. A. Tretyakov Phys. Rev. E 72, 066606 (2005).

16 W. H. Weber and G. W. Ford, Phys. Rev. B 70, 125429 (2004).

17 A. F. Koenderink and A. Polman, Phys. Rev. B 74, 033402 (2006);

18 A. Alù and N. Engheta, Phys. Rev. B 74, 205436 (2006).

19 M. C. K. Wiltshire, E. Shamonina, I. R. Young, and L. Solymar, J. Appl. Phys. 95, 4488 (2004).

${ }^{20}$ M. Meier and A. Wokaun, Opt. Lett. 8, 581 (1983).

21 For Drude models with $\epsilon_{b} \neq 1, \gamma=0, \kappa$ is reduced by $\left(3 \epsilon_{m} /\left(\epsilon_{b}+2 \epsilon_{m}\right) \approx 0.66\right.$ for Ag in glass.

22 We fit optical data in 23 using $\epsilon_{\text {metal }}=\epsilon_{b}-\eta \omega_{p}^{2} /(\omega(\omega+i \gamma)$. For silver, $\epsilon_{b}=5.45, \eta=0.73, \omega_{p}=1.72 \cdot 10^{16} \mathrm{~s}^{-1}$ and $\gamma=$ $8.35 \cdot 10^{13} \mathrm{~s}^{-1}$.

23 Handbook of optical constants of solids, edited by E. D. Palik (Academic, Orlando, FL, 1985). 